\title{
Ganzheitliche Palliativmedizin am Lebensende
}

Eine gut durchgeführte Palliativpflege am Ende des Lebens kann Schmerzen und andere Beschwerden lindern, seelischen Beistand leisten und eine gute Lebensqualität erhalten. Die Weltgesundheitsorganisation (WHO) empfiehlt daher, die Palliativmedizin zu einem festen Bestandteil der Gesundheitsversorgung zu machen und allen Patienten, die an einer zum Tode führenden Erkrankung leiden, anzubieten.

n dem aktuellen systematischen Review wurden insgesamt 14 Studien berücksichtigt. In sieben Studien wurden Modelle zu chronischen Erkrankungen (Demenz, Multiple Sklerose, chronische Herzinsuffizienz, HIV/AIDS und fortgeschrittene chronische Erkrankungen) evaluiert, in vier Studien ging es um die integrative Pflege in der Onkologie, drei Studien beschäftigten sich mit malignen und chronischen Erkrankungen, die zum Tode führten. Die meisten Studien kamen aus Großbritannien (sechs), zwei aus Spanien, zwei aus den Niederlanden und jeweils eine aus Italien, Deutschland, Norwegen und Frankreich.

Die Ergebnisse von 13 der 14 Studien erbrachten eindeutige Vorteile für die Anwendung ganzheitlicher Palliativmedizin: bessere Symptomkontrolle, eine geringere Belastung für die Pflegenden, Verbesserungen hinsichtlich Kontinuität und Koordination der Pflege, seltenere Klinikeinweisungen und Kostenersparnis. Auch verstarben die Patienten häufiger dort, wo sie es wünschten. Keine einzige der untersuchten Studien konnte ein in allen Punkten optimal funktionie-

\section{Schrumpfende Gesellschaften im internationalen Vergleich}

Ein Forschungsprojekt des FraunhoferInstituts für System- und Innovationsforschung ISI hat untersucht, wie in Deutschland, China, Italien, Japan und der Schweiz mit bereits spürbaren sowie zu erwartenden gesellschaftlichen Folgen des demografischen Wandels umgegangen wird. Dabei spielt nicht nur die Frage der Alterung von Gesellschaften, sondern auch ihre mögliche Schrumpfung eine wichtige Rolle. Das Ziel des vom Bundesministerium für Bildung und Forschung (BMBF) geförderten Projektes ist es, aus den Erfahrungen anderer Länder zu lernen, die ebenfalls vom demografischen Wandel betroffen sind. Hieraus wurden Empfehlungen und Vorschläge für künftige BMBF-Forschungsprojekte an der Schnittstelle der Themenbereiche "Mensch-Technik-Interaktion" und „Demografischer Wandel" abgeleitet.

Schrumpfende Gesellschaften im Vergleich. Maßnahmen gegen die Folgen des demografischen Wandels. Fraunhofer-Institut, Juli 2016

\section{Hier steht eine Anzeige.}

\author{
偤 Springer
}

